NIST Technical Note 2156

\title{
Metrological Traceability Frequently Asked Questions and NIST Policy
}

\author{
Antonio Possolo \\ Sally S. Bruce \\ Robert L. Watters, Jr.
}


NIST Technical Note 2156

\title{
Metrological Traceability Frequently Asked Questions and NIST Policy
}

\author{
Antonio Possolo \\ Statistical Engineering Division \\ Information Technology Laboratory \\ Sally S. Bruce \\ Standards Coordination Office \\ Robert L. Watters, Jr. \\ Office of Reference Materials (Retired Associate) \\ Material Measurement Laboratory
}

This publication is available free of charge from: https://doi.org/10.6028/NIST.TN.2156

May 2021

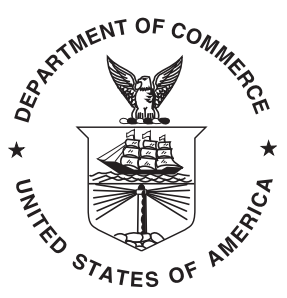

U.S. Department of Commerce Gina M. Raimondo, Secretary

National Institute of Standards and Technology James K. Olthoff, Performing the Non-Exclusive Functions and Duties of the Under Secretary of Commerce for Standards and Technology \& Director, National Institute of Standards and Technology 
Certain commercial entities, equipment, or materials may be identified in this document in order to describe an experimental procedure or concept adequately. Such identification is not intended to imply recommendation or endorsement by the National Institute of Standards and Technology, nor is it intended to imply that the entities, materials, or equipment are necessarily the best available for the purpose.

National Institute of Standards and Technology Technical Note 2156

Natl. Inst. Stand. Technol. Tech. Note 2156, 42 pages (May 2021)

CODEN: NTNOEF

This publication is available free of charge from: https://doi.org/10.6028/NIST.TN.2156 


\begin{abstract}
The NIST policy on metrological traceability (NIST P 5800.00, which became effective on May 31st, 2019) is transcribed and supplemented with a review of relevant terminology and with a list of frequently asked questions and answers. This list updates and amplifies a similar list originally prepared by a team organized and led by Richard Kayser, which was published in the World Wide Web, and comprises questions about: metrological traceability in general; establishing metrological traceability; NIST and NIST's role in metrological traceability; NIST products and services; and international aspects of traceability.
\end{abstract}

\title{
Key words
}

accreditation; calibration; categorical; fitness for purpose; measurand; measurement; nominal; ordinal; qualitative property; quantitative property; reference material; Standard Reference Data; Standard Reference Instrument; Standard Reference Material; traceability 


\section{Table of Contents}

1 Introduction 4

2 History 4

3 NIST Policy 5

3.1 Purpose 5

3.2 Policy 5

$\begin{array}{llr}4 & \text { Terminology } & 7\end{array}$

5 Frequently Asked Questions $\quad 14$

5.1 Questions about Metrological Traceability in General 14

$\begin{array}{ll}\text { 5.1.1 What is metrological traceability? } & 14\end{array}$

$\begin{array}{ll}\text { 5.1.2 What is measurement? } & 14\end{array}$

5.1.3 Is it correct to say that measurements, measuring instruments, or standards are traceable? $\quad 15$

5.1.4 Are traceable measurement results fit for purpose? 16

5.1.5 Is it correct to say that an organization is traceable? 16

5.1.6 Who is responsible for supporting claims of traceability? 16

5.1.7 What do I need to do to support a claim of traceability? 16

5.1.8 Who is responsible for assessing the validity of claims of traceability? 17

5.1.9 What should I look for in a valid claim of traceability?

5.1.10 Are counted quantities traceable? 17

5.1.11 Are qualitative properties traceable? 19

5.1.12 Does the redefinition of the SI impact traceability? 20

5.2 Questions about Establishing Metrological Traceability 20

5.2.1 What is involved in establishing metrological traceability? 20

5.2.2 Who is responsible for establishing the traceability of measurement results?

5.2.3 Does metrological traceability need to be reexamined periodically? What are the criteria for judging the integrity of the metrological traceability chain?

5.2.4 Is a NIST Test Report Number necessary and/or sufficient evidence of traceability?

5.2.5 To establish the traceability of a measurement result for a particular measurand, is it also necessary to establish the traceability of all other values of measured properties associated with the measurand, especially those that might contribute in a relatively minor way? For example, when making a dimensional measurement, do the contemporaneous measurements of barometric pressure, humidity, and temperature need to be traceable to the International System of Units (SI)? 
5.2.6 Is a consensus value metrologically traceable that has been derived from traceable measurement results produced by the participants in an interlaboratory study or key comparison?

5.3 Questions about NIST and NIST's Role in Metrological Traceability

5.3.1 What is NIST?

5.3.2 What is NIST's role in metrological traceability? 23

5.3.3 What is meant by the phrase "traceable to NIST"?

5.3.4 I want my measurement results to be traceable to NIST. What do I have to do?

5.3.5 How does NIST establish the traceability of its own measurement results?

5.3.6 How does NIST support its own claims of traceability?

5.3.7 Does NIST assure the traceability of its own measurement results?

5.3.8 Does NIST certify the traceability of measurement results other than its own?

5.3.9 Given that NIST operates the National Voluntary Laboratory

Accreditation Program (NVLAP), does this mean that NIST stands behind claims of traceability made by NVLAP-accredited labs?

5.4 Questions about NIST Products or Services

5.4.1 Does NIST have publications that explain what traceability is and how to achieve it?

5.4.2 What products and services does NIST provide in support of customers seeking to establish traceability?

5.4.3 If I have an instrument or artifact calibrated at NIST, does that make my measurement results traceable to those of NIST?

5.4.4 If I purchase an SRM, does that make my measurement results traceable to NIST?

5.4.5 If I purchase a NIST Traceable Reference Material ${ }^{(\mathrm{CM})}\left(\mathrm{NTRM}^{(\mathrm{CM})}\right)$, does that make my measurement results traceable to those of NIST? 28

5.4.6 NIST data are often used in claims of traceability to NIST. Under what conditions can one consider NIST data to be "specified references" in the sense that this term is used in Defining metrological traceability and in Specified reference?

5.4.7 Can organizations claim traceability to NIST by having individual instrument components calibrated at NIST? If so, is that the same as sending the entire instrument to NIST for calibration?

5.4.8 How should customers claim traceability to NIST if NIST needs to use two or more of its own standards to calibrate the customer instrument, standard, or artifact?

5.4.9 Can customers claim traceability to NIST for measurement results associated with something that NIST cannot calibrate directly? 
5.5.1 What is the CIPM MRA and what does it have to do with traceability?

5.5.2 If a laboratory establishes traceability of its measurement results to standards maintained by National Metrology Institutes (NMIs) that is a signatory to the CIPM MRA, does that mean that that laboratory's measurement results are traceable to standards maintained by other signatory NMIs?

5.5.3 What are laboratory accreditation MRAs and what do they have to do with traceability?

5.5.4 Because NIST's National Voluntary Laboratory Accreditation Program (NVLAP) is a signatory to multiple MRAs with other accreditation bodies, does that mean NIST stands behind claims of traceability made by any laboratory accredited by any other MRA signatory? 


\section{Introduction}

NIST maintains a policy on metrological traceability and supplements it with informal clarifications, supporting materials, and answers to questions that NIST staff and customers of NIST measurement services ask frequently.

Section 3 transcribes the corresponding directive, NIST P 5800.00, which came into effect on May 31st, 2019.

Section 4 reviews the meaning of specific terms used in discussions of traceability and of how it is established, as well as general terms used in metrology that are often employed in such discussions.

Section 5 lists questions that are frequently asked about traceability, and provides answers to them, often accompanied by some discussion of relevant concepts or associated practices.

These questions pertain to: metrological traceability in general; establishing metrological traceability; NIST and NIST's role in metrological traceability; NIST products and services; and international aspects of metrological traceability, in particular the relationship between the provisions of the CIPM Mutual Recognition Arrangement (MRA) [1] and traceability.

\section{History}

The original version of this collection of Frequently Asked Questions and Answers (FAQ) about traceability was prepared by a team convened and led by Rich Kayser (in his role at the time, of Director of the NIST Technology Services), at the request of Karen Brown, who served as acting Director of NIST during 2000-2001. This team included Gordon Day, Dennis Friday, Al Parr, Don Sullivan, and Dennis Swyt, among others.

That FAQ was deployed on the Web as Supplementary Materials Related to NIST Policy on Metrological Traceability. Subsequently, Bob Watters (formerly Director of the Office of Reference Materials) maintained the website and replied to inquiries about it.

Belanger et al. [2] provide an overview of the early development of the concept of metrological traceability at NIST. The longstanding commitment of NIST to traceability as a foundation stone in the reliability and overall quality of measurement is amply illustrated in numerous contributions to different areas of measurement, including [3], [4], [5], [6], [7], and [8], among others. 


\section{$3 \quad$ NIST Policy}

The following two subsections transcribe verbatim (except for a few corrections of typos in the punctuation) the contents of the PURPOSE and POLICY sections of NIST Directive P 5800.00, Metrological Traceability, which became effective on May 31st, 2019.

\subsection{Purpose}

The mission of the National Institute of Standards and Technology (NIST) is to promote U.S. innovation and industrial competitiveness by advancing measurement science, standards, and technology in ways that enhance economic security and improve our quality of life. To help meet the measurement and standards needs of U.S. industry and the Nation, NIST provides calibrations, standard reference materials, standard reference data, test methods, proficiency evaluation materials ${ }^{1}$, tools that facilitate the evaluation of measurement uncertainty ${ }^{2}$, measurement quality assurance programs, and laboratory accreditation services that assist customers in establishing traceability of measurement results.

Metrological traceability ${ }^{3}$ requires the establishment of an unbroken chain of calibrations to specified reference measurement standards: typically national or international standards, in particular realizations of the measurement units of the International System of Units (SI). NIST assures the traceability to the SI, or to other specified standards, of measurement results that NIST itself provides, either directly or through an official NIST program or collaboration. Other organizations are responsible for establishing the traceability of their own results to national reference standards maintained by NIST or to other specified reference standards. NIST has adopted this policy statement to document the NIST role with respect to traceability.

\subsection{Policy}

To support the conduct of its mission and to ensure that the use of its name, products, and services is consistent with its authorities and responsibilities, NIST:

1. Adopts for its own use and recommends for use by others the definition of traceability provided in the most recent version of the International vocabulary of metrology - Basic and general concepts

\footnotetext{
${ }^{1}$ Homogeneous materials or artifacts that are used to test and evaluate the measurement performance and fitness for purpose of measuring systems (VIM §3.2) or measurement procedures (VIM §2.6).

${ }^{2}$ NIST Uncertainty Machine (https : / / uncertainty.nist.gov) and NIST Consensus Builder (https://consensus.nist.gov), with user's manuals available online, and supported by peer-reviewed publications: Lafarge \& Possolo (NCSLI Measure Journal of Measurement Science 10(3): 20-27); Koepke et al. (2017, Metrologia 54(3): S34-S62, DOI 10.1088/1681-7575/aa6c0e).

${ }^{3}$ The abbreviated term "traceability" is sometimes used to mean "metrological traceability" as well as other concepts, such as "sample traceability" or "document traceability" or "instrument traceability" or "material traceability," where the history ("trace") of an item is meant. Therefore, the full term of "metrological traceability" is preferred if there is any risk of confusion (VIM §2.41, Note 8).
} 
and associated terms $\left(\mathrm{VIM}^{4} \S 2.41\right)$ : "property of a measurement result whereby the result can be related to a reference through a documented unbroken chain of calibrations, each contributing to the measurement uncertainty."

2. Establishes metrological traceability to the SI, or to other specified standards, of its own measurement results and of measurement results provided to customers in official reports and certificates of NIST calibrations, reference materials, and other measurement services that operate in accordance with the NIST Quality System for Measurement Services.

3. Asserts that providing support for a claim of metrological traceability of a measurement result is the responsibility of the provider of that result, whether that provider is NIST or another organization, and that assessing the validity of such a claim is the responsibility of the user of that result.

4. Communicates, especially where claims expressing or implying the contrary are made, that NIST does not define, specify, assure, or certify metrological traceability of measurement results other than those that NIST itself provides, either directly or through an official NIST program or collaboration.

5. Emphasizes that traceability alone does not signify or guarantee fitness for purpose, because this typically requires that the uncertainty associated with a measured value or calibration be sufficiently small to satisfy a particular measurement need.

6. Collaborates on the development of standard definitions, interpretations, and recommended practices with organizations that have authority and responsibility for variously defining, specifying, assuring, or certifying metrological traceability.

7. Develops and disseminates technical information on traceability, provides tools and expertise supporting the evaluation of measurement uncertainty, and conducts coordinated outreach programs on issues of traceability and related requirements.

8. Assigns responsibility for oversight of implementation of the NIST policy on metrological traceability to the NIST Measurement Services Council (NMSC).

\footnotetext{
${ }^{4}$ Joint Committee for Guides in Metrology (2012, 3rd Edition, International Bureau of Weights and Measures (BIPM)). The VIM is freely available online at https://jcgm.bipm.org/vim/en/.
} 


\section{Terminology}

For definitions and explanations of metrological terms and other relevant terminology in general use, refer to the following resources:

- NIST Quality Manual for Measurement Services (NIST-QM-I)

- Metrological Tools for the Reference Materials and Reference Instruments of the NIST Material Measurement Laboratory [9]

- IUPAC Green Book [10]

- IUPAP Red Book [11]

- ISO Guide 30: Reference materials - Selected terms and definitions [12]

- International Vocabulary of Metrology [JCGM 200:2012]

NIST aims to remain generally consistent with national and international documentary guides, including the International Union of Pure and Applied Chemistry (IUPAC)'s "Gold" [14] and "Green" [10] Books and the International Union of Pure and Applied Physics (IUPAP)'s "Red Book" [11], but reserves the right to define and use metrological terminology in ways that it deems best, that are consistent with NIST's mission, and that serve U.S. national needs. NIST disseminates such definitions and usage in documents such as this one, and in other NIST publications and documents supporting the NIST Quality Management System [9].

Since the following terms may be used throughout this document in ways that are specific to NIST, their meaning is reviewed and clarified as follows:

Assuring metrological traceability is to provide support for the claim of traceability of a particular measurement result. Organizations that have the authority and responsibility for variously defining, specifying, assuring, or certifying traceability include any regulatory agency, standards developing organization, accreditation body, trade association or the like, which, by law or mutual agreement, is assigned or takes on authority and responsibility for some aspect of defining, specifying, assuring, or certifying traceability.

Certified Reference Material (CRM) is a reference material (RM) characterized by a metrologically valid procedure for one or more specified properties, accompanied by an RM certificate that provides the value of the specified property, its associated uncertainty, and a statement of metrological traceability (ISO Guide 30, 2.1.2) [ISO 30:2015].

Certified values delivered by a CRM are believed by NIST to be [9]:

- characteristic of the property(ies) specified (measurand), 
- characteristic of the material at some defined minimum sample size (homogeneity),

- stable (for some defined period when properly stored and handled),

- accurate (unbiased within a specified level-of-confidence interval),

- metrologically traceable (to a higher-order reference system), and

- documented well enough to provide users with confidence that the certified value is fit for the purpose(s) specified in the documentation supplied to the customer.

Certifying metrological traceability is to attest formally that traceability has been established for a specific measurement result.

Defining metrological traceability is to state or set forth authoritatively the (operational) meaning of the term "metrological traceability."

The primary purpose of the NIST Policy on Traceability (http://www . nist.gov/traceability), transcribed in 3.2 above, is to state the NIST role with respect to traceability. The Policy presents the definition of measurement traceability used by NIST, and clarifies the roles of NIST and others in achieving traceability of measurement results for measurements both internal and external to NIST.

The NIST Policy on Traceability also addresses the role of NIST in providing its customers with the tools they need (a) to assist them in establishing traceability of their measurement results, and (b) to assess the claims of traceability made by others.

This is achieved directly through the provision of NIST measurementrelated products and services, through collaboration with relevant organizations, through development and dissemination of technical information on traceability, and through conducting coordinated outreach programs.

Merely using an instrument or artifact calibrated at NIST is not enough to make the measurement result traceable to reference standards developed and maintained by NIST. As detailed in 5.1.7, to establish traceability, the provider of a measurement result must document the measurement process or system used to establish the claim and provide a description of the chain of calibrations that were used to establish a connection to a specified reference: the instrument or artifact calibrated at NIST then being one of the links in this chain.

Internal measurement assurance program is a NIST program that lends credibility to measurement results (measured values and associated uncertainties) produced in the topical focus area of the program, for which traceability is to be established. Such program typically involves monitoring the performance (e.g., stability, reproducibility) of the measuring instruments, standards of reference, or measurement 
systems within the scope of the program, before and after they are characterized, calibrated, or used to obtain the traceable measurement results.

Laboratory accreditation is a procedure whereby an authoritative body formally recognizes that a laboratory is competent to carry out specific tasks. Accreditation does not itself qualify the laboratory to approve any particular product. However, accreditation may be relevant to approval and certification authorities when they decide whether or not to accept data produced by a given laboratory in connection with their own activities [ISO/IEC 17011:2017] [ISO/IEC 17025:2017] [ISO 17034:2016]. NIST's National Voluntary Laboratory Accreditation Program (NVLAP) provides third-party accreditation to testing and calibration laboratories in response to legislative actions or requests from government agencies or privatesector organizations.

National or international standards are measurement standards recognized as references for assigning values to other standards of the property of interest, either at a national level, or by an international agreement.

NIST Calibration Reports and Reports of Special Tests record the result of a calibration or of a special test. NIST calibrates instruments and devices that are metrologically suitable as reference or transfer standards.

Special Tests are so designated for one or more of the following reasons: (1) the specific type of calibration is seldom requested, thus precluding the maintenance of a large statistical base for characterizing the measurement process; (2) the test requested is unique; or (3) the service is still under development - meaning that the measurement or calibration methods are still being perfected, or that some of the quality-control documentation is still incomplete.

These reports state one or more property values and their associated uncertainties, and confirm that the necessary procedures have been carried out to ensure their validity and traceability. The results of calibrations and tests performed by NIST apply only to the specific instrument or standard at the time of test unless otherwise clearly stated.

\section{NIST Certificates of Analysis, Reference Material Information Sheets, and} Reports of Investigation accompany reference materials with NIST certified material property values, or with non-certified values only. From 1987 until the publication of NIST Special Publication 260-136 (2020 Edition) [9], NIST noncertified values were called "NIST Reference Values" if an (incomplete) uncertainty estimate was associated with the value, or "NIST Information Values" if they were not qualified with an uncertainty statement.

The following examples illustrate the types of documents that accompany reference materials currently available for sale by the Office of Reference Materials. These documents also confirm that the necessary procedures have been carried out to ensure the validity and traceability of the results that they report, as applicable [9] [12] [18] [17]. 
- NIST Standard Reference Material 3128, Lead (Pb) Standard Solution, is accompanied by a certificate of analysis stating the certified value of the mass fraction of lead in the solution;

- The Information Sheet for NIST Reference Material 8666, Ginger (Zingiber officinale) Extract, includes the non-certified values of the mass fraction of several gingerols in the extract;

- The Report of Investigation for Reference Material 8851, Zeolite A, provides a (non-certified) reference value for the mass fraction of sodium in the material, and an information value for the refractive index of the material.

NIST Reference Materials (RMs) deliver property values for established measurands that are fit for the materials' intended uses, including between-method harmonization, within-method precision assessment, and process stability assessment. RMs are issued as "RM 8xxx", where the " $\mathrm{xxx}$ " is a unique series of three digits. These materials and values have historically been described in a "Report of Investigation" rather than in a Certificate or Certificate of Analysis. For RMs developed after the publication of NIST Special Publication 260-136 (2020 Edition) [9], these documents are called "Reference Material Information Sheets." However, some older materials still available for sale are accompanied by a Report of Investigation (for example, for the aforementioned Reference Material 8851, Zeolite A.

NIST Standard Reference Data (SRD) provide information whose reliability has been critically evaluated and that: (i) is related to a measurable physical, or chemical, or biological property of a substance or system of substances of known composition and structure; (ii) comprises measurable characteristics of a physical artifact or artifacts; (iii) specifies engineering properties or performance characteristics of a system; or (iv) serves to calibrate or characterize the performance of a detection or measurement system, or to interpolate or extrapolate measurement results (Standard Reference Data Act, U.S.C. Title 15, Chapter 7A, Section 290(a))

NIST Standard Reference Instruments (SRIs) are devices that have been designed and validated by NIST and that are available for purchase by users of our measurement services, similarly to SRMs. SRIs whose certificates include a statement of metrological traceability give these customers the ability to obtain traceable results when metrological traceability cannot be adequately established through reference materials or other calibrated measuring instruments. Some SRIs allow customers to realize a unit of the International System of Units (SI) (i.e., experimentally to establish the value consistently with the definition of the SI unit), particularly when they exploit the advantages of the new SI (refer to 5.1.12). SRIs are issued as "SRI 6xxx" where "xxx" denotes a unique sequence of three digits.

NIST Standard Reference Materials ${ }^{\circledR}$ (SRMs $^{\circledR}$ ) are CRMs (ISO Guide 30, 2.1.2) [ISO 30:2015] that deliver at least one certified property value that is metrologically traceable to an internationally recognized higher-order reference system, such as the SI, 
that are deemed by NIST to be suitable for providing unbiased calibration and/or validation of measurement procedures, and that have been produced consistently with [9] and [ISO 17034:2016].

SRMs may also deliver non-certified information. The certified and non-certified values are described in separate sections in the SRM's documentation: "Certificate" for physical or engineering performance properties, and "Certificate of Analysis" for specific chemical properties. The certificate that reports the results of the characterization indicates the intended use of the material, explains how to use it, and states the date when the certification expires.

SRMs may undergo recertification, or their assigned values or uncertainties may be corrected, which triggers an update to the corresponding certificate. For example, the original certificate for NIST SRM 342a, Nodular Cast Iron - Chip Form, was issued in 1999, and then reissued in 2019 following a reevaluation of the expanded uncertainties associated with the certified values.

SRMs are issued as "SRM xxxx" where "xxxx" typically denotes a number between 1 and 5999, with a suffix that is either alphabetic or designates a lot number, indicating re-issues. NOTE: "Standard Reference Material" and "SRM" are trademarks registered to NIST by the United States Patent and Trademark Office.

NIST Traceable Reference Materials ${ }^{(\mathrm{CM})}\left(\mathrm{NTRMs}^{(\mathrm{CM})}\right)$ are Certified Reference Materials (CRMs) that are certified by NIST in collaboration with a secondary standards producer, with a well-defined metrological traceability linkage to existing SRMs or primary standards. This linkage is established via criteria and protocols defined by NIST to meet the needs of the community served. Reference materials producers adhering to these requirements can use the "NTRM" certification mark, NTRM ${ }^{(\mathrm{CM})}$. The certified values are described in a "Certificate of Traceability." Two NTRM programs have been established: an ongoing specialty gas program [19] [20], and a spectrophotometric filter program [21] that ended in 2005.

Official NIST program or collaboration is a NIST program or collaboration that has been officially approved by NIST management, in which NIST formally assures or certifies traceability of the results of measurements other than those that NIST itself provides. One of these programs is the NIST Traceable Reference Material Program for Gas Standards, established in 1992 in partnership with the U.S. Environmental Protection Agency (EPA) and specialty gas companies to increase the availability of standard gas mixtures with certified composition that support the "Emissions Trading" provision of the 1990 Clean Air Act.

Proficiency evaluation materials are either homogeneous materials or stable artifacts that are used to test and evaluate the measurement performance of different measuring systems when they are employed in specific tasks.

Provider of a measurement result is the individual or organization that supplies a measurement result for use, whose metrological traceability is being asserted. 
Reference material (RM) is a material, sufficiently homogeneous and stable with respect to one or more specified properties, which has been established to be fit for its intended purpose in a measurement procedure. RM is a generic term, and the properties RMs deliver can be quantitative or qualitative, e.g., identity of substances or biological species [ISO 30:2015] (adapted).

Research Gas Mixtures (RGMs) are specialty gas CRMs produced as single units in collaboration with an industrial or government agency partner. RGMs are certified using the same protocols used for batch-certified NIST gas SRMs. RGMs are identified using the cylinder's identification code. The certified values are described in a Certificate of Traceability.

Specified reference is shorthand for "specified reference measurement standard," where: (i) "specified" means that it has been explicitly set forth in supporting documentation; and (ii) "reference measurement standard" refers to a measurement standard generally of the highest metrological quality available at a given location or in a given organization, used as a reference in the assignment of values by this organization. A national measurement standard is a standard recognized by a national authority to serve in a state or economy as the basis for assigning values to quantitative or qualitative properties, in other standards of the property of interest. An international measurement standard is a standard recognized by signatories of an international agreement and intended to serve worldwide.

Specifying metrological traceability is to assert a requirement for traceability in a given situation.

Stated or reported uncertainties are expressions of measurement uncertainty explicitly set forth in supporting documentation to qualify a measured value that were evaluated in accordance with the GUM [JCGM 100:2008] and its supplements, or, when reported by NIST, also in accordance with the NIST Quality Manual, and with NIST Technical Notes 1297 [23] and 1900 [24].

Test methods are logical sequences of operations, described generically, used to characterize the performance of measurements or specified technical procedures for performing a test [ISO/IEC 2:2004].

Unbroken chain of calibrations or comparisons is a complete, explicitly described, and documented series of calibrations that successively link a measurement result (measured value and associated uncertainty) to the values and uncertainties of each of the intermediate reference standards, and to the highest reference standard to which traceability for the measurement result is claimed.

A calibration is an operation that, under specified conditions: in a first step, establishes a relation between the property values with their associated uncertainties delivered by measurement standards and corresponding instrumental indications with their associated measurement uncertainties; and, in a second step, uses 
this information to derive measurement result from an instrumental indication together with its associated uncertainty.

User of a measurement result is the individual or organization that takes delivery of, and is the intended user of, a measurement result for which metrological traceability is being asserted. 


\section{Frequently Asked Questions}

\subsection{Questions about Metrological Traceability in General}

\subsubsection{What is metrological traceability?}

The definition of metrological traceability that has achieved global acceptance in the metrology community is stated in the International vocabulary of metrology - Basic and general concepts and associated terms (VIM) [JCGM 200:2012, 2.41]: property of a measurement result whereby the result can be related to a reference through a documented unbroken chain of calibrations, each contributing to the measurement uncertainty.

It is important to note that traceability is the property of the result of a measurement, not of an instrument or calibration report or laboratory. It is not achieved by following any one particular procedure or using particular equipment. Merely having an instrument calibrated, even by NIST, is not enough to make the measurement result obtained using that instrument traceable to realizations of the appropriate SI unit or other specified references. The measurement system by which values and uncertainties are transferred must be understood clearly and be under appropriate quality control.

\subsubsection{What is measurement?}

The NIST Quality Manual for Measurement Services (NIST-QM-I) defines "measurement" and "measurement uncertainty" as follows:

MEASUREMENT: Experimental or computational process that, by comparison with a standard, produces an estimate of the true value of a property of a material or virtual object or collection of objects, or of a process, event, or series of events, together with an evaluation of the uncertainty associated with that estimate, and intended for use in support of decision-making.

MEASUREMENT UNCERTAINTY: Doubt about the true value of the measurand that remains after making a measurement; measurement uncertainty is described fully and quantitatively by a probability distribution on the set of values of the measurand; at a minimum, it may be described summarily and approximately by a quantitative indication of the dispersion (or scatter) of such distribution.

For information and guidance on evaluating and expressing measurement uncertainty, refer to NIST Technical Notes 1297 [23] and 1900 [24].

- The property intended to be measured (measurand) may be qualitative (for example, the identity of the nucleobase at a particular location of a strand of DNA), or quantitative (for example, the mass concentration of 25 -hydroxyvitamin $\mathrm{D}_{3}$ in NIST SRM 972a, Level 1, whose certified value is $28.8 \mathrm{ng} \mathrm{mL}^{-1}$ ). The measurand may also be an ordinal property (for example, the Rockwell $C$ hardness of a material), or a function whose values may be quantitative (for example, relating the response of a force transducer to an 
applied force) or qualitative (for example, the provenance of a glass fragment determined in a forensic investigation).

- A measurement standard is a realization or embodiment of the definition of a quantity, including a statement of the value of the quantity and the associated measurement uncertainty (VIM 5.1). This realization may be provided by a measuring system (VIM 3.2), a material measure (VIM 3.6), or a reference material (ISO Guide 30, 2.1.1 [ISO 30:2015]). The aforementioned "comparison with a standard" may be direct (for example, using a comparator for the dimensions of gauge blocks), or indirect, via a calibrated instrument (for example, using a force transducer that has been calibrated at NIST).

\subsubsection{Is it correct to say that measurements, measuring instruments, or standards are traceable?}

Only measurement results are traceable. Refer to 5.1.1

The results of calibrations and tests performed by NIST are intended solely for the use of the organization requesting them, and apply only to a particular device or specimen at the time of its test.

The results shall not be used to indicate or imply that they are applicable to other similar items, in particular in commercial advertisements. In addition, such results must not be used to indicate or imply that NIST approves, recommends, or endorses the manufacturer, the supplier, or the user of such devices or specimens, or that NIST in any way "guarantees" the performance of items after calibration or test.

A NIST customer or collaborator may state as a fact that calibrations provided by NIST are used in part to establish metrological traceability to the SI or other specified reference using the results of NIST measurements.

NIST declares it to be in the national interest that NIST should maintain an impartial position with respect to any commercial product. Advertising the findings on a single instrument, device, or artifact could be misinterpreted as an indication of performance of other instruments, devices, or artifacts of identical or similar type.

There will be no objection, however, to a statement that the manufacturer's primary standards have been periodically calibrated by NIST, if this is actually the case, or that the customer might arrange to have NIST calibrate the item purchased from the manufacturer.

NIST does not approve, recommend, or endorse any product or proprietary material. No reference shall be made to NIST or to reports or results furnished by NIST in any advertising or sales promotion, which would indicate or imply that NIST approves, recommends, or endorses any product or proprietary material, or whose purpose is to promote the use or purchase of an advertised product owing to NIST test reports or results. 


\subsubsection{Are traceable measurement results fit for purpose?}

Not necessarily or automatically, because traceability imposes no restrictions on the size of the uncertainty associated with a measured value, while fitness for purpose typically involves some requirement about how large measurement uncertainty may be in order for the measured value to be usable in a particular application. "Metrological traceability of a measurement result does not ensure that the measurement uncertainty is adequate for a given purpose or that there is an absence of mistakes" (VIM 2.41, Note 5).

Similarly, when a user measures a property of a NIST SRM whose value is certified, and determines that the measured value does not differ significantly from the certified value, this does not establish that the user's measurement result is fit for purpose, and fulfills only one of the requirements to establish traceability to a standard of reference maintained by NIST, or to the SI (refer to 5.1.1 and to 5.1.7).

\subsubsection{Is it correct to say that an organization is traceable?}

Neither organizations nor laboratories can be traceable. Only measurement results can be traceable.

\subsubsection{Who is responsible for supporting claims of traceability?}

The provider of the result of a measurement is responsible for supporting its claim of the traceability of that result or value. This is the case whether that provider is NIST or another organization.

\subsubsection{What do I need to do to support a claim of traceability?}

To support a claim of traceability, the provider of a measurement result must document the measurement process or system used to establish the claim and provide a description of the chain of calibrations that were used to establish a connection to a specified reference. There are several common elements to all valid statements or claims of traceability:

- a clearly defined, particular property (quantitative or qualitative) that has been measured;

- a complete description of the measurement system or working standard used to perform the measurement;

- a stated measurement result, which comprises a measured value and a documented evaluation of the associated measurement uncertainty;

- a complete specification of the reference at the time the measurement system or working standard was compared to it; 
- an internal measurement assurance program for establishing the status of the measurement system or working standard at all times pertinent to the claim of traceability;

- an internal measurement assurance program for establishing the status of the specified reference at the time that the measurement system or working standard was compared to it.

An internal measurement assurance program may be very simple or rather complex, the level or rigor to be determined depending on the level of uncertainty at issue and what is needed to demonstrate its credibility. Users of a measurement result are responsible for determining what is adequate to meet their needs.

Refer to Checklist for Traceability through Calibration for additional guidance.

\subsubsection{Who is responsible for assessing the validity of claims of traceability?}

The end-user of the result of a measurement, and conformity assessment bodies are responsible for assessing the validity of a claim of traceability. However, as explained in the answer to Question 5.1.6 above, the provider is responsible for providing the necessary information that the user assesses.

\subsubsection{What should I look for in a valid claim of traceability?}

The answer to Question 5.1.7 explains what the provider of a measurement result should do to support a claim of traceability. As a user, you should look for these elements. Refer to Checklist for Traceability through Calibration for additional guidance.

\subsubsection{Are counted quantities traceable?}

Since counting is measuring, the question naturally arises about the meaning of traceability for counts, for example when one counts the number of neutrophils among 100 white blood cells in a patient's sample (a typical practice for differential leukocyte counts).

The conventional position has been to say that counts are traceable to the unit 1 , which is the neutral element in the SI. Referring to counts, and somewhat cryptically, the 9th edition of the SI Brochure (§2.3.3) states that "formal traceability to the SI can be established through appropriate, validated measurement procedures" [26].

Establishing traceability for counts requires further elaboration. Their traceability cannot be to the SI, but will have to be to other references. Counting involves two kinds of standards: one standard defines the entities that are being counted (and distinguishes them from those other entities that are not to be counted); another standard that serves to assign a value to the count. 
The first standard plays the role of what, in zoology and botany, is called the holotype (of a species): in the present context, it is the paradigmatic or ideal instantiation of what is being counted - the typical neutrophil, when counting white blood cells, or the typical photon, when counting photons. The same as with biological species, some diversity often needs to be accommodated, because neutrophils are not exact copies of one another, and photons, too, vary according to their wavelength.

In zoology, for example, the diversity corresponding to differences due to gender, may be accommodated by designating allotypes: this makes it possible to recognize both mallard drakes and hens as members of the same species, Anas platyrhynchos, even though they look quite different from one another. The diversity of neutrophils may have to be accommodated by formulating a standard that comprises a sufficiently diverse collection of images of neutrophils, supplemented with descriptions of identifying attributes (for example, having a multi-lobed nucleus, intra-cellular granules, or a characteristic response to specific staining agents).

The second standard needed for counting finitely many entities is the unique subset of the consecutive positive integers including 1 and its successors, in the sense of Peano's axioms [27] that can be put into one-to-one correspondence with the elements of the set whose elements are being counted. The value of the count is the largest integer in the standard. If no such subset of the positive integers exists, then we say that the count is 0 .

In the case of a differential white blood cell count that yields 63 neutrophils out of 100 white blood cells, saying that the measured value 63 is traceable to the unit 1 is as trivial and as unproductive as saying that $63 \times 1=63$. The measurement result needs to specify what is being counted, and in addition ought to include also an evaluation of measurement uncertainty: using the Poisson model, a clinical laboratory technician doing the count manually might then report having found 63 neutrophils give or take 8 neutrophils. This margin of uncertainty accounts for sampling variability, as different blood smears from the same person typically will not yield exactly the same differential leukocyte count, and possibly accounts also for mere counting error.

Claiming that counts are traceable to the SI is too much of a simplification because it neglects the fact that counting inextricably involves the definition of what is being counted, and the standard that underlies this definition is not part of the SI.

This limitation applies also to quantities like chemical amounts: when one says that a baby aspirin contains $0.45 \mathrm{mmol}$ of acetylsalicyclic acid, one is indeed expressing a count, of molecules in this case, qualified with a statement of what is being counted, molecules of acetylsalicyclic acid in one baby aspirin pill, but the definition of aspirin is not within the scope of the SI. On the other hand, $0.45 \mathrm{mmol}$ of acetylsalicyclic acid, with a relative molecular mass of $180.159 \mathrm{mg} / \mathrm{mmol}$ is equivalent to $81 \mathrm{mg}$ of acetylsalicyclic acid, which may be traceable to the kilogram. 


\subsubsection{Are qualitative properties traceable?}

Qualitative (also called categorical) properties, which are nominal or ordinal [28], may be measurands. The values assigned to them should be qualified with uncertainty evaluations. Measurements of qualitative properties may also be called identifications, classifications, etc., depending on the context, yet without suggesting that all identifications, classifications, etc. necessarily are measurements. The values assigned to qualitative properties may be traceable to a reference that is recognized and accepted by the relevant community, but they will not be traceable to the SI.

A nominal property is a kind of qualitative property whose values partition the set of entities that possess it into subsets such that all the entities in the same subset have the same value of the property. (Being a partition means that different subsets have no elements in common, and their union is the set that has been partitioned.) For example, the set of the nucleobases in a strand of DNA can be partitioned according to their chemical identity: adenine, cytosine, guanine, or thymine.

The values of a nominal property are the names of the subsets in that partition. The values of nominal properties can be compared only by establishing equality or difference. For example,a chemical element either is or is not an alkali metal; a specific tiger has the same defining traits as other tigers, and these traits differentiate all tigers from animals of other species in the genus Panthera.

An ordinal property is a quality whose values may be ordered in a substantively meaningful way, but for which neither arithmetic differences nor ratios are meaningful, even when the values are expressed using numbers. For example, the Mohs hardness of a mineral [29, Chapter 2], or the strength of a hurricane according to the Saffir-Simpson Hurricane Wind Scale.

The expression of the uncertainty associated with a value assignment to a nominal or ordinal property may be a (discrete) probability distribution over the set of values that the property possibly can take. For example, one may say that the nucleobase at a particular locus in a DNA strand is adenine with $93 \%$ probability, cytosine with $2 \%$ probability, guanine with $4 \%$ probability, or thymine with $1 \%$ probability.

Or this uncertainty may be expressed only qualitatively, on an ordinal scale. For example, the preparation of NIST SRM 3246 involved identifying the species of the plant, Ginkgo biloba, the material originates from. The identification was based on Sanger sequencing of the plant's DNA, and the associated uncertainty was expressed on an ordinal scale comprising these levels: Ambiguous, Confident, Very Confident, and Most Confident. According to Beauchamp et al. [9], identities can be certified only when the assessed confidence is at its highest level.

Establishing traceability for an assignment of value to a nominal property, for example that the nucleobase at a particular position of a strand of DNA is adenine, involves comparison with a standard for adenine relevant to how the nucleobase is identified, which may involve quantitative attributes, for example the mass spectrum as specified in the NIST Chemistry WebBook (Standard Reference Database 69). 
Establishing traceability to the Mohs hardness scale for minerals involves performing a scratch test of the mineral specimen of interest, and involving a realization of the scale (a collection of specimens of talc, gypsum, calcite, fluorite, apatite, orthoclase, quartz, topaz, corundum, and diamond), to determine which minerals in the realization of the scale the specimen of interest scratches, and which scratch the specimen.

\subsubsection{Does the redefinition of the SI impact traceability?}

The redefinition of the SI approved by the 26th General Conference of Weights and Measures on November 16, 2018, generally facilitates the establishment of traceability for several units in the SI.

For example, prior to the redefinition, the international prototype of the kilogram (IPK) was used only once every 50 years, which made it difficult for the BIPM ("Bureau International des Poids et Mesures", or International Bureau of Weights and Measures) to maintain traceability of its working mass standards to the kilogram [30]. Also, establishing traceability for mass standards with masses much smaller than $1 \mathrm{~kg}$ required a chain of multiple calibrations, whose uncertainty increased in proportion to the number of mass comparisons involved. The new SI makes realizations of the unit of mass and of convenient sub-multiples of it readily accessible in the laboratory and on the factory floor, via the Kibble balance [31].

Similarly, a cell containing a vapor of cesium-133 atoms excited to a Rydberg state can be used as a sensor to measure electric field strength [32] [33], which is intrinsically traceable to the SI in the sense that it enables measurements depending only on the electric dipole moment of these atoms and on the Planck constant, which now has a fixed value.

\subsection{Questions about Establishing Metrological Traceability}

\subsubsection{What is involved in establishing metrological traceability?}

Per the VIM definition, metrological traceability is established through "an unbroken chain of calibrations, each contributing to the measurement uncertainty." In practical terms, this means having in place, for each link in the chain:

- A clearly defined particular property that has been measured;

- A complete description of the measurement system or working standard used to perform the measurement;

- A stated measurement result comprising a measured value and a documented evaluation of the associated measurement uncertainty;

- A complete specification of the references used for calibrating the measurement system or working standard when it was most recently calibrated; 
- An internal measurement assurance program for establishing the status of the measurement system or working standard at all times pertinent to the claim of traceability;

- An internal measurement assurance program for establishing the status of the reference used to calibrate the measurement system or working standard when it was most recently calibrated.

An internal measurement assurance program may be very simple or rather complex: its level or rigor will depend on the level of uncertainty at issue and on what is needed to demonstrate its credibility. Users of a measurement result are responsible for determining what is adequate to meet their needs and to ensure that the measurement results are fit for purpose. For information and guidance on evaluating and expressing measurement uncertainty, refer to NIST Technical Notes 1297 [23] and 1900 [24].

\subsubsection{Who is responsible for establishing the traceability of measurement results?}

The provider of the result of a measurement is responsible for establishing the traceability of this result. This is the case whether that provider is NIST or another organization.

\subsubsection{Does metrological traceability need to be reexamined periodically? What are the criteria for judging the integrity of the metrological traceability chain?}

Yes, traceability does need to be reexamined periodically, and the criteria for assessing the integrity of the traceability chain involve consideration of: the measurement requirements; the needs of the client; the dependability of the equipment and standards; the environmental effects; etc. For more details, refer to [34].

\subsubsection{Is a NIST Test Report Number necessary and/or sufficient evidence of trace- ability?}

Test report numbers issued by NIST are intended to be used solely for administrative purposes. Although they are often used to identify documents that support traceability claims, test report numbers themselves do not address the issues listed in the answer to Question 5.2.1, and should neither be used nor required as the sole proof of traceability. 


\subsubsection{To establish the traceability of a measurement result for a particular mea- surand, is it also necessary to establish the traceability of all other values of measured properties associated with the measurand, especially those that might contribute in a relatively minor way? For example, when making a di- mensional measurement, do the contemporaneous measurements of baro- metric pressure, humidity, and temperature need to be traceable to the In- ternational System of Units (SI)?}

As a general principle, the definition of metrological traceability in the VIM suggests that it is necessary to establish the traceability of all input quantities in a measurement model whose output is the measured value whose traceability is being ascertained. A measurement model defines the way in which a measured value depends upon the values of specified inputs. For example, a dimensional measurement result may depend upon barometric pressure, humidity, and temperature, if either the values or the uncertainties surrounding the values of these inputs contribute significantly to the value or to the uncertainty of the measurement result.

From a practical viewpoint, the contribution that an input to a measurement model makes to the measurement result is significant if a change in the value or uncertainty of the input induces a change in the significant figures that should be reported for the value or for the uncertainty of the measurement result. When this is the case, traceability for the measurement of the relevant inputs should be established at levels of uncertainty that render the measurement result fit for purpose.

\subsubsection{Is a consensus value metrologically traceable that has been derived from trace- able measurement results produced by the participants in an interlaboratory study or key comparison?}

The consensus value may be construed as the output quantity in a measurement model as defined in the GUM [22, Equation (1)], the measurement results produced by the participants as the inputs, and the data reduction procedure that produces the consensus value as the function that maps inputs to the output, in that same model. Since this function and the inputs determine the output, and the inputs are traceable, then according to the answer to Question 5.2.5 the consensus value will be traceable as well. If it is intended that the consensus value output of an interlaboratory study be traceable, then the protocols and reporting requirements of the study must be designed such that all the supporting evidence for the traceability of the inputs is documented as listed in the answer to Question 5.2.1 is provided.

\subsection{Questions about NIST and NIST's Role in Metrological Traceability}

\subsubsection{What is NIST?}

The National Institute of Standards and Technology (NIST) is a non-regulatory agency of the U.S. Department of Commerce. Its role as the National Metrology Institute (NMI) 
for the United States was established by Congress in 1901. As such, NIST has the responsibility "to develop, maintain, and retain custody of the national standards of measurement, and to provide the means and methods for making measurements consistent with those standards; to assure the compatibility of United States national measurement standards with those standards; and to assure the compatibility of United States national measurement standards with those of other nations." [15 U.S.C. 271]

The job of NIST in this particular is twofold: to ensure U.S. national standards are accurate realizations of the units of the international system of units (SI), and to transfer the values of those standards to the U.S. measurement system through calibrations, reference materials, and other measurement services. Direct customers of NIST's measurement services take advantage of the opportunity to tie their internal measurement standards to NIST standards and hence, to the SI units. These customers, in turn, use their standards to provide measurement services to their customers, to meet regulatory requirements, and to provide quality assurance in their manufacturing processes. NIST also provides laboratory accreditation services to testing and calibration laboratories through its National Voluntary Laboratory Accreditation Program (NVLAP). (Refer also to the answer to Question 5.4.4.)

\subsubsection{What is NIST's role in metrological traceability?}

NIST has several roles in traceability:

- First, to provide practical access to the seven base units of the International System of Units (SI) - second, meter, kilogram, ampere, kelvin, mole, and candela -, by realizing and disseminating those units through measurement services;

- Second, and similarly, to provide access to what are in effect U.S. national standards of various other measurable properties of economic importance to the United States; and

- Third, to collaborate on the development of standard definitions, interpretations, and recommended practices with organizations that have authority and responsibility for variously defining, specifying, assuring, or certifying traceability.

\subsubsection{What is meant by the phrase "traceable to NIST"?}

Metrological traceability is a property of a measurement result by which that result is related to specified reference standards, not to institutions. Accordingly, the phrase "traceable to NIST" can be meaningful only if it is used as shorthand for "metrologically traceable to NIST's practical realization of the definition of a measurement unit" [JCGM 200:2012, 2.43], or for "traceable through NIST to the SI or to other specified reference standard." 


\subsubsection{I want my measurement results to be traceable to NIST. What do I have to do?}

To achieve traceability of measurement results to standards maintained by NIST, you need to reference your measurement results through an unbroken chain of calibrations, including determining the uncertainties at each step, to NIST standards as the specified references. These references may be, for example, standards developed and maintained by NIST: broadcast signals controlled or monitored by NIST (such as standard time and frequency signals), NIST Standard Reference Materials (SRMs), NIST-Traceable Reference Materials (NTRMs), or those NIST Standard Reference Instruments (SRIs) whose certificates include a statement of metrological traceability. The chain of calibrations may be short if the user has instruments or artifacts calibrated by NIST or acquires standards from NIST and references measurement results to those. Or it may be long if the user references other calibrations in a chain of calibrations back to stated references developed and maintained by NIST. Also see Question 5.1 .7 above and Checklist for Traceability through Calibration for additional guidance.

\subsubsection{How does NIST establish the traceability of its own measurement results?}

In general, NIST establishes the traceability of its own measurement results via an unbroken chain of calibrations, including evaluating measurement uncertainty at each step, to specified references. In the case of the SI base units, the ultimate stated reference is the definition of the units, including associated procedures to realize them, as established by the General Conference on Weights and Measures (CGPM) and specified in the SI Brochure [26]. The same process also applies in the case of derived units, which are formed as products of powers of the base units according to the algebraic relations linking the quantities concerned. For other measurement quantities, the reference may be a national standard defined de facto by a conventionally stated method.

\subsubsection{How does NIST support its own claims of traceability?}

NIST documents the process by which it establishes traceability of its own measurement results as part of the NIST Quality System for Measurement Services [35]. In accordance with the Quality System, NIST maintains current technical descriptions of (a) the measurement facility, system, or methods; (b) the procedures for conduct of the measurement(s); (c) the analysis of uncertainty of the measurement results; and (d) the procedures for statistical control of the measurement process. This documentation includes a description of how traceability is/was achieved.

\subsubsection{Does NIST assure the traceability of its own measurement results?}

According to the NIST policy on traceability, NIST establishes the traceability of the results of its own measurements and of results provided to customers in NIST calibration reports and measurement certificates, in particular in Certificates of Analysis that accompany NIST SRMs. 
For example, the Certificate of Analysis for NIST SRM 3161a Tin (Sn) Standard Solution (Lot No. 140917) states that "the measurand is the total concentration of tin, expressed as mass fraction and the certified value is metrologically traceable to the SI unit for mass." The Certificate also indicates that "This SRM can be used to establish traceability of the results of tin measurements to NIST measurement results and standards. One approach is to calibrate analytical instruments or procedures for the determination of tin using standards whose values are traceable to the certified value of tin in this SRM."

NIST provides a Practical Tool for Establishing Traceability in Chemical Measurements implemented in an Excel Workbook, including instructions for use and an example data set.

Although the measurement results in a calibration or measurement certificate can be considered to be certified by NIST as being traceable to NIST reference standards at the time the measurements were performed at NIST, no such certification extends automatically to measurement results that a user will obtain using an instrument calibrated by NIST or using a NIST Standard Reference Material.

The certificate of each Standard Reference Material specifies the conditions under which NIST stands behind its measurement results after the SRM has left NIST. The customer must have an appropriate internal measurement assurance program in place to assure the traceability of measurements of the same materials, or of measurements made using those materials as calibrants [34].

\subsubsection{Does NIST certify the traceability of measurement results other than its own?}

NIST only certifies the traceability of measurement results that NIST itself provides, either directly or through an official NIST program or collaboration. NIST cannot be responsible for claims of traceability made by others since the process of demonstrating traceability requires that most of the steps be taken at the site of whoever is claiming traceability. NIST has no control over this process and no direct involvement in the dayto-day activities at the site. (See Section 6, Checklist for Traceability through Calibration for supporting a claim of traceability through calibration for an illustration of activities to be performed at a customer facility, as opposed to activities performed at a reference laboratory.)

\subsubsection{Given that NIST operates the National Voluntary Laboratory Accreditation Program (NVLAP), does this mean that NIST stands behind claims of trace- ability made by NVLAP-accredited labs?}

No, laboratory accreditation, whether conducted by NIST/NVLAP or any other recognized accreditation body, is a finding of a laboratory's competence and capability to provide technically sound and appropriate measurement services within their scope of accreditation. Embedded in the process is an evaluation of the lab's ability to achieve and maintain traceability for the accredited services. 
Accreditation to ISO/IEC Guide 17025 [ISO/IEC 17025:2017] determines that a laboratory has all of the necessary facilities, equipment, standards, procedures, uncertainty analyses, personnel, etc., which make it capable of providing traceable measurement results. Laboratory accreditation does not speak to the specifics of any individual measurement result but to the overall capability of a lab to provide the service. NIST experts often participate in the accreditation process, but again, the end result is a finding of competence and capability only. All calibration and test reports must declare the source of traceability, and it is the responsibility of the user to determine which source is suitable for their needs.

\subsection{Questions about NIST Products or Services}

\subsubsection{Does NIST have publications that explain what traceability is and how to achieve it?}

Yes, NIST has several series of publications that are relevant to traceability. Some of these publications appear in the list of references that begins on Page 35.

\subsubsection{What products and services does NIST provide in support of customers seek- ing to establish traceability?}

NIST provides a range of products and measurement services in support of customers seeking to establish traceability of their measurement results. These include instrument calibrations, Standard Reference Materials (SRMs), Standard Reference Instruments (SRIs), and under certain circumstances Standard Reference Data (SRD) (refer to Question 5.4.6). NIST also provides measurement services to State and local governments responsible for marketplace transactions that involve measurements (weights and measures).

NIST's Calibration Program, Standard Reference Materials Program, and the Weights and Measures Program make up the largest integrated national measurement transfer system in the world. The Standard Reference Data Program augments this system by providing scientists, engineers, and the general public with access to critically evaluated data necessary to perform state-of-the-art research and development.

Through these four programs, NIST disseminates expert metrology guidance and the measurement products and services developed in the NIST Laboratories to the industrial and scientific communities, federal agencies, and state and local governments.

Calibration laboratories and testing facilities may be accredited by the National Voluntary Laboratory Accreditation Program (NVLAP), which is administered by NIST. Leaman and Hackett [36] describe the basic procedures and general accreditation requirements of NVLAP. A participating laboratory may voluntarily take steps to improve or assess its measurement process.

NIST Laboratories also provide a range of training courses and workshops on measurement practices. Topics covered include precision thermometry, temperature measure- 
ment by radiation thermometry, laser measurements, gage block calibration, time and frequency fundamentals, dimensional metrology, pressure, etc.

For general information about measurement units, refer to The NIST Reference on Constants, Units, and Uncertainty, and to SI Units. For information and guidance on evaluating and expressing measurement uncertainty, refer to NIST Technical Notes 1297 [23] and 1900 [24], and to the NIST Uncertainty Machine [37].

\subsubsection{If I have an instrument or artifact calibrated at NIST, does that make my mea- surement results traceable to those of NIST?}

Merely having an instrument or artifact calibrated at NIST is not enough to make the measurement result obtained by the user of the instrument traceable to reference standards developed and maintained by NIST. To establish traceability of the user's measurement result to such reference standards, there must be a documented, unbroken chain of calibrations where each measured value must be qualified by a statement of the associated uncertainty. The measurement system by which values are transferred must be clearly understood and under control. The establishment dates and details of each link in the chain must also be provided.

\subsubsection{If I purchase an SRM, does that make my measurement results traceable to NIST?}

The purchase and use of an SRM does not automatically make the customer's measurement results traceable to reference standards developed and maintained by NIST. The intended purpose of most NIST certified reference materials, including SRMs, is to provide higher-order calibration and validation materials to secondary standard producers and customers intending to produce metrologically traceable in-house calibrants and control materials [9]. However, NIST SRMs are only one of the elements that enable a claim of traceability for a measurement result.

A claim of traceability to reference standards developed and maintained by NIST can be asserted by proper use of appropriate SRMs and reference to the certified values and uncertainties provided by a NIST Certificate of Analysis. This Certificate contains a statement of the intended use of the SRM (NOTE: NIST cannot foresee all possible uses for a specific SRM and recognizes that defensible assertions can be made for alternative use).

In general, the Certificate also contains a disclaimer for improper handling of a material. It is up to the user to document appropriate storage and preservation of a material. An SRM certificate also contains an expiration date. It is inappropriate to use a material past its expiration date to establish a traceability claim. Finally, it is up to the user to ensure that the Certificate of Analysis in their possession is valid and up-to-date. Users of NIST SRMs should register themselves as the end-user so that NIST can notify them when an updated certificate is available. 


\subsubsection{If I purchase a NIST Traceable Reference Material ${ }^{(\mathrm{CM})}\left(\mathrm{NTRM}^{(\mathrm{CM})}\right)$, does that make my measurement results traceable to those of NIST?}

The same as with SRMs, Question 5.4.4, the purchase and use of an NTRM does not automatically make the customer's measurement results traceable to reference standards developed and maintained by NIST. However, NTRMs can be used to make a claim of traceability to NIST in the same manner as SRMs, with reference to the certified values and uncertainties on an NTRM Certificate of Analysis.

\subsubsection{NIST data are often used in claims of traceability to NIST. Under what con- ditions can one consider NIST data to be "specified references" in the sense that this term is used in Defining metrological traceability and in Specified reference?}

NIST provides several data services that are critical to the measurement infrastructure of the United States and the world. These services include:

- Standard Reference Data, that is, data that have been evaluated for validity by knowledgeable experts in the particular field with stated uncertainties;

- Values of physical constants measured by NIST researchers and published in the peer-reviewed literature with stated uncertainties;

- Measurements of properties that result from various NIST research programs, which may or may not have complete uncertainty evaluations;

- Collections of unevaluated data compiled by NIST scientists for various research and development purposes.

Great effort is made to describe clearly the type of data in NIST electronic and printed data collections. Usually, data from NIST are qualified with uncertainties evaluated in accordance with the GUM.

When data are evaluated, the scientific basis for such evaluation is well documented and the data are labeled as NIST Standard Reference Data. The suitability of using these data as "stated references" depends on the type of collection (Standard Reference Data, values of physical constants, etc.), the date of the most recent evaluation or compilation, and the direct relevance of the data to the physical measurement for which traceability is needed. In particular, if the data are not part of a NIST compilation of evaluated data, then care should be taken to assess the expressed uncertainty, and the literature checked for additional or updated values, for comparison. 


\subsubsection{Can organizations claim traceability to NIST by having individual instrument components calibrated at NIST? If so, is that the same as sending the entire instrument to NIST for calibration?}

If the claim of traceability to reference standards developed and maintained by NIST is supported by an appropriate measurement assurance process and uncertainty analysis, the claim may be valid: refer to Questions 5.1.1 and 5.1.7 for details. Also, the measurement process and uncertainty analysis must show how the basic component measurements are combined and translated into a complete calibration for the instrument in question. Whether it is the same as sending the complete instrument in depends on whether the organization combines the results using exactly the same measurement model and methods that NIST would use in calibrating the entire instrument. However, this is ordinarily not the case.

\subsubsection{How should customers claim traceability to NIST if NIST needs to use two or more of its own standards to calibrate the customer instrument, standard, or artifact?}

NIST may use several internal standards to calibrate or validate its own measurement process or system. The measurement results with associated uncertainties are links in the traceability chain. The customer should claim traceability to the NIST system comprising the individual standards and should indicate who combined the data using what algorithm. Uncertainties should be documented, calculated, and reported accordingly.

\subsubsection{Can customers claim traceability to NIST for measurement results associated with something that NIST cannot calibrate directly?}

Yes, under certain circumstances. If well-accepted, scientifically sound and appropriate measurement equipment, practices, and procedures are used; if measurement values and uncertainties are calculated or otherwise established according to well-established protocols; and if the uncertainties are within accepted norms (both low and high) for the measurement application; then the customer's measurement result might be considered traceable to NIST.

For example, there are many measurable quantities for which NIST does not offer calibration services, but that are derived by combining other quantities for which NIST does provide calibration services. Torque is one of these quantities. NIST does not offer a calibration service for torque. It does, however, offer services for the component measurement quantities (force, length, etc.) that are needed to derive torque, according to the well-accepted definition of torque. By performing the steps necessary to achieve traceability to NIST of the individual component measurement results when making a particular measurement of torque, it is possible to claim traceability of the corresponding measurement result to NIST.

The uncertainty of the measurement result must first be calculated (again using a well- 
established methodology, such as that in NIST Technical Notes 1297 [23] or 1900 [24]) using the uncertainties associated with the individual component measurement quantities. This uncertainty must be at (or above) a realistic level of uncertainty consistent with the sophistication of the measurement model and other practical and established considerations.

As another example, NIST does provide calibration services for voltage, but only at discrete values of voltage. If an organization wants to establish traceability to NIST at other values of voltage, it must develop an interpolation or extrapolation procedure and a protocol for doing this. Additional measurement uncertainties will result. The resulting statement of traceability must incorporate these additional uncertainties, which must again be realistic and within expected norms.

As a third example, NIST may not offer any calibration service or SRM for a particular quantity, but may provide measurement protocols and advice, along with expected norms for levels of uncertainty that can be achieved according to the protocol. Under these circumstances, an organization may again claim traceability of its measurement results to NIST if the protocols (and built-in checks/tests) are followed, and the claimed uncertainties are within the expected norms. For more on the "Elements of Traceability" refer to [34].

\subsection{Questions about the MRA and Traceability}

\subsubsection{What is the CIPM MRA and what does it have to do with traceability?}

The CIPM Mutual Recognition Arrangement (MRA) was drawn up by the International Committee of Weights and Measures (CIPM) under the authority given to it in the Metre Convention, for signature by directors of member National Metrology Institutes (NMIs) [ISO/IEC 17025:2017].

The principal objectives of the MRA are: (i) to perform measurement comparisons that establish degrees of equivalence of national measurement standards maintained by NMIs; (ii) to provide for the mutual recognition of calibration reports and measurement certificates issued by NMIs; and (iii) to provide a secure technical foundation for wider agreements related to international trade, commerce, and regulatory affairs.

The mutual recognition of calibration and measurement certificates requires that each NMI participate regularly in the activities of the International Bureau of Weights and Measures (BIPM) (including key measurement comparisons) and have a suitable way of assuring quality in the results of its measurement services.

The results of the key measurement comparisons and specific statements of the calibration and measurement capabilities (CMCs) of each signatory NMI are entered in an MRA database originally developed by NIST and now maintained by the Bureau of International Weights and Measures at the Key Comparison Database.

While NIST recognizes the validity of the other signatories' certificates, such recognition does not mean that measurement results traceable to any other signatory are therefore 
traceable to NIST. The burden of establishing traceability of its measurement results to NIST, and of supporting an associated claim of traceability, is on the individual NMI providing the service.

In March, 2015, the CIPM decided (Decision CIPM/104-26) that delta value isotope ratio measurements that cannot presently be made traceable to the SI should be made traceable to materials recognized as International Standards. Brand et al. [38] provide a list of certified reference materials that should be used to identify accepted references for delta value isotope ratio traceability statements.

\subsubsection{If a laboratory establishes traceability of its measurement results to standards maintained by National Metrology Institutes (NMIs) that is a signatory to the CIPM MRA, does that mean that that laboratory's measurement results are traceable to standards maintained by other signatory NMIs?}

While signatory NMIs (including NIST) recognize the validity of other signatories' measurement and calibration certificates under the MRA, such recognition does not mean that measurement results obtained by one signatory NMI are automatically traceable to stated references developed and maintained by any other signatory NMI.

However, users of measurement results, who may be either commercial or regulatory entities, may well decide that sufficient evidence exists under the MRA to provide mutually acceptable traceability of these results to the standards and measurements of two or more participating NMIs. Such evidence may include comparable claims of calibration and measurement capabilities of the NMIs for a particular measurement or calibration service, coupled with satisfactory performance on a key comparison of the same measurement or standard by each of the NMIs.

\subsubsection{What are laboratory accreditation MRAs and what do they have to do with traceability?}

Laboratory accreditation MRAs are established among accreditation bodies, domestically and internationally, for the purpose of recognizing the equivalence of their respective accreditation systems. To be invited to sign an MRA, each accreditation body must undergo an evaluation by its peers to determine its capability and competence to meet documented requirements for the operation of such bodies. Included is the ability of the accreditation body to accredit laboratories to the requirements of the internationally recognized standards ISO/IEC Guide 17025 [ISO/IEC 17025:2017] or ISO Guide 17034 [ISO 17034:2016].

The purpose of these MRAs is to provide the means by which users of calibration and testing services can have confidence in the calibration and test reports issued by a laboratory that has been accredited by any of the accreditation body signatories to the MRA. The goals are to reduce or eliminate redundant audits by having one evaluation of a laboratory satisfy the needs of the user community and to have the results of calibrations or tests accepted across borders. 
By signing the arrangement, each signatory is obligated to promote the acceptance of the results of calibrations or tests conducted by the accredited laboratories of any other signatory to the MRA as being technically equivalent to its own. This does not mean that signatory accreditation bodies accept or promote the acceptance of claims of traceability to sources other than NIST as being equal to traceability to standards maintained by NIST. All calibration and test reports must declare the source of traceability and it is the responsibility of the user to determine which source is suitable for its needs. Other tools, such as the CIPM MRA and the databases resulting from this MRA, are meant to assist the users in making this decision. (See Questions 5.5.1 and 5.5.2 above.)

\subsubsection{Because NIST's National Voluntary Laboratory Accreditation Program (NVLAP) is a signatory to multiple MRAs with other accreditation bodies, does that mean NIST stands behind claims of traceability made by any laboratory ac- credited by any other MRA signatory?}

As previously stated, laboratory accreditation, whether conducted by NVLAP or any other recognized accreditation body, is a finding of a laboratory's competence and capability to provide technically sound and appropriate measurement services within their scope of accreditation. As a signatory to MRAs operated under the auspices of the Asia Pacific Accreditation Cooperation (APAC), the InterAmerican Accreditation Cooperation, and the International Laboratory Accreditation Cooperation (ILAC), NVLAP promotes international acceptance of test, calibration and inspection reports and other documents issued by laboratories and inspection bodies accredited by signatories to the MRAs. All calibration and test reports must declare the source of traceability and it is the responsibility of the user to determine which source is suitable for its needs. 


\section{Checklist for Traceability through Calibration}

A high-level checklist for traceability through calibration is as follows:

Element 1(performed at customer facility, responsibility of customer)

- Identify appropriate transfer instrument/standard/system, based on consultation with reference laboratory

- Set up a measurement assurance program to characterize the transfer instrument/standard/system, establishing measurement assurance charts (indicating values and uncertainties associated with the instrument/standard/system)

- Ship transfer instrument/standard/system to calibration laboratory

\section{Element 2 (performed at reference laboratory, responsibility of reference labo- ratory)}

- After unpacking the transfer instrument/standard/system and inspecting for damage, identify an appropriate reference standard that is already part of a measurement assurance program at the reference laboratory (and that can provide measurement results traceable to national primary standards for the quantity in question)

- Perform the calibration of the customer's transfer instrument, standard, or system, preparing a calibration report with the measurement results (measured values and associated measurement uncertainties), and including a statement of metrological traceability that includes the details of the reference standard.

- Ship the transfer instrument/standard/system back to the customer along with the calibration report.

\section{Element 3 (performed at customer facility, responsibility of customer)}

- After unpacking the transfer instrument/standard/system and inspecting for damage, carry out measurements according to the same process and procedures used in the measurement assurance program discussed in Element 1

- Analyze the new measurement assurance chart to evaluate the condition of the transfer instrument/standard/system with respect to any change in measurement characteristics (compared with prior to being shipped to the reference laboratory) (NOTE: use the original values, not the new calibrated values, when making this evaluation)

- Establish the appropriate values and uncertainties to use with the newly-calibrated transfer instrument/standard/system, modifying and annotating the control charts accordingly but continuing the measurement assurance program 


\section{Element 4 (performed at customer facility, responsibility of customer)}

- Just prior to performing a measurement using the transfer instrument/standard/system, characterize it using the measurement assurance program to verify its integrity and performance

- Use the transfer instrument/standard/system to perform a measurement, the result of which is desired to be traceable to the reference standard at the reference laboratory

- Evaluate the uncertainty associated with this measurement result, taking into account the uncertainty stated in the calibration report, and specifying how the uncertainty components were evaluated (Type A or Type B)

- Just after performing the measurement using the transfer instrument/standard/system, re-characterize it using the same measurement assurance program to re-verify its integrity and performance (giving reassurance that its characteristics and performance during the measurement were likely as expected)

- Formally document all of the steps in these four Elements to the degree necessary, depending on the importance of the particular measurement result, or to the satisfaction of the requiring organization

\section{Acknowledgments}

The authors are much indebted to Steven Choquette, Charles Ehrlich, Katherine Sharpless, and Gregory Strouse, all from NIST, for numerous comments, corrections, and suggestions that greatly improved this contribution. 


\section{References $^{5}$}

[1] Comité International des Poids et Mesures (CIPM) (1999) Mutual Recognition of National Measurement Standards and of Calibration and Measurement Certificates Issued by National Metrology Institutes (Bureau International des Poids et Mesures (BIPM), Pavillon de Breteuil, Sèvres, France). Technical Supplement revised in October 2003 Available at www.bipm.org/en/cipm-mra/.

[2] Belanger B, Rasberry S, Garner E, Brickencamp C, Ehrlich C (2001) Traceability: An evolving concept. A Century of Excellence in Measurements, Standards, and Technology, ed Lide DR (National Institute of Standards and Technology, Gaithersburg, MD), NIST Special Publication, Vol. 958, pp 167-171. A Chronicle of Selected NBS/NIST Publications 1901-2000.

[3] Swyt DA, Phillips SD, Palmateer JW (2001) Developments at NIST on traceability in dimensional measurements. Recent Developments in Traceable Dimensional Measurements, eds Decker JE, Brown N International Society for Optics and Photonics (SPIE), Vol. 4401, pp 245-252. https://doi.org/10.1117/12.445626

[4] Pratt JR, Kramar JA, Newell DB, Smith DT (2005) Review of SI traceable force metrology for instrumented indentation and atomic force microscopy. Measurement Science and Technology 16(11):2129-2137. https://doi.org/10.1088/09570233/16/11/002

[5] Orji NG, Dixson RG, Cordes AM, Bunday BD, Allgair JA (2011) Measurement traceability and quality assurance in a nanomanufacturing environment. fournal of Micro/Nanolithography, MEMS, and MOEMS 10(1):1-7. https://doi.org/ 10.1117/1.3549736

[6] Karam LR (2014) Measurement traceability in medical physics. Fournal of Medical Physics 39:1-3. https://doi.org/10.4103/0971-6203.125470

[7] Lombardi MA, Novick AN, Neville-Neil G, Cooke B (2016) Accurate, traceable, and verifiable time synchronization for world financial markets. fournal of Research of the National Institute of Standards and Technology 121:436-463. https://doi.org/ 10.6028/jres.121.023

[8] T Hedberg J, Helu M, Feeney AB, Krima S (2020) Recommendations on Ensuring Traceability and Trustworthiness of Manufacturing-Related Data (National Institute of Standards and Technology, Gaithersburg, MD). https://doi.org/ 10.6028/NIST.AMS.300-10. NIST Advanced Manufacturing Series 300-10

[9] Beauchamp CR, Camara JE, Carney J, Choquette SJ, Cole KD, DeRose PC, Duewer DL, Epstein MS, Kline MC, Lippa KA, Lucon E, Phinney KW, Possolo A, Sharpless

\footnotetext{
${ }^{5}$ The numbers between square brackets following each reference are clickable links that point and lead to the pages in this document where the references are cited.
} 
KE, Sieber JR, Toman B, Winchester MR, Windover D (2020) Metrological Tools for the Reference Materials and Reference Instruments of the NIST Materials Measurement Laboratory. NIST Special Publication 260-136 (2020 Edition) (National Institute of Standards and Technology, Gaithersburg, MD). https://doi.org/10.6028/NIST.SP.260136-2020

$[7,9,10,11,19$,

27]

[10] Cohen E, Cvitaš T, Frey J, Holmström B, Kuchitsu K, Marquardt R, Mills I, Pavese F, Quack M, Stohner J, Strauss H, Takami M, Thor A (2008) Quantities, Units and Symbols in Physical Chemistry (IUPAC \& RSC Publishing, Cambridge,UK), 3rd Ed. IUPAC Green Book.

[11] Cohen ER, Giacomo P (1987) Symbols, units, nomenclature and fundamental constants in physics. International Union of Pure and Applied Physics (IUPAP), Commission C2 - SUNAMCO, Institute of Physics, London, UK. Document IUPAP25 (SUNAMCO 87-1, "Red Book"), Reprinted from Physica A: Statistical Mechanics and its Applications 146 (1987) 1-68, 2010 Reprint.

[12] ISO (2015) Reference materials - Selected terms and definitions (International Organization for Standardization (ISO), Geneva, Switzerland), Third Ed. ISO Guide 30:2015(E).

[13] Joint Committee for Guides in Metrology (2012) International vocabulary of metrology - Basic and general concepts and associated terms (VIM) (International Bureau of Weights and Measures (BIPM), Sèvres, France), 3rd Ed. BIPM, IEC, IFCC, ILAC, ISO, IUPAC, IUPAP and OIML, JCGM 200:2012 (2017 version with minor corrections and informative annotations) Available at https://jcgm.bipm.org/ $\mathrm{vim} / \mathrm{en} /$.

[14] International Union of Pure and Applied Chemistry (2012) Compendium of Chemical Terminology ("Gold Book") (International Union of Pure and Applied Chemistry, Research Triangle Park, NC). https://doi.org/10.1351/goldbook. Available at goldbook.iupac.org/

[15] ISO/IEC (2017) Conformity assessment - Requirements for accreditation bodies accrediting conformity assessment bodies (International Organization for Standardization (ISO), Geneva, Switzerland), Third Ed. ISO/IEC 17011:2017(EN).

[16] ISO/IEC (2017) General requirements for the competence of testing and calibration laboratories (International Organization for Standardization (ISO), Geneva, Switzerland), Third Ed. ISO/IEC Standard 17025:2017.

$[9,26,30,31]$

[17] ISO (2016) General requirements for the competence of reference material producers (International Organization for Standardization (ISO), Geneva, Switzerland), Third Ed. ISO Guide 17034:2016. 
[18] ISO (2015) Reference materials - Contents of certificates, labels and accompanying documentation (International Organization for Standardization (ISO), Geneva, Switzerland), Third Ed. ISO Guide 31:2015.

[19] Guenther FR, Dorko WD, Miller WR, G R (1996) The NIST Traceable Reference Material Program for Gas Standards. NIST Special Publication 260-126 (National Institute of Standards and Technology, Gaithersburg, MD). https://doi.org/ 10.6028/NIST.SP.260-126

[20] Dorko WD, Kelley ME, Guenther FR (2015) The NIST Traceable Reference Material Program for Gas Standards. NIST Special Publication 260-126 Rev2013 (National Institute of Standards and Technology, Gaithersburg, MD). https://doi.org/ 10.6028/NIST.SP.260-126rev2013

[21] Travis JC, Smith MV, Rasberry SD, Kramer GW (2000) Technical Specifications for Certification of Spectrophotometric NTRMs. NIST Special Publication 260-140 (National Institute of Standards and Technology, Gaithersburg, MD). https://doi.org/ 10.6028/NIST.SP.260-140

[22] Joint Committee for Guides in Metrology (JCGM) (2008) Evaluation of Measurement Data - Guide to the Expression of Uncertainty in Measurement (International Bureau of Weights and Measures (BIPM), Sèvres, France). BIPM, IEC, IFCC, ILAC, ISO, IUPAC, IUPAP and OIML, JCGM 100:2008, GUM 1995 with minor corrections Available at www.bipm.org/en/publications/guides/gum.html.

[23] Taylor BN, Kuyatt CE (1994) Guidelines for Evaluating and Expressing the Uncertainty of NIST Measurement Results. NIST Technical Note 1297 (National Institute of Standards and Technology, Gaithersburg, MD). Available at https : //physics.nist.gov/Pubs/guidelines/TN1297/tn1297s.pdf. [12, 14, 21, 27, 30]

[24] Possolo A (2015) Simple Guide for Evaluating and Expressing the Uncertainty of NIST Measurement Results (National Institute of Standards and Technology, Gaithersburg, MD). https://doi.org/10.6028/NIST.TN.1900. NIST Technical Note 1900 [12, 14, 21, 27, 30]

[25] ISO/IEC (2004) Standardization and related activities - General vocabulary (International Organization for Standardization (ISO), Geneva, Switzerland). ISO/IEC Guide 2:2004(EN).

[26] BIPM (2019) The International System of Units (SI) (International Bureau of Weights and Measures (BIPM), Sèvres, France), 9th Ed. Available at https://www. bipm . org/en/publications/si-brochure/.

[27] Mints GE (2018) Peano axioms. Encyclopedia of Mathematics (Springer \& European Mathematical Society). Last modified on 1 December 2018 Available at https: //www. encyclopediaofmath.org/index.php/Peano_axioms.

[28] Agresti A (2019) An Introduction to Categorical Data Analysis (John Wiley \& Sons, Hoboken, NJ), 3rd Ed. 
[29] Klein C, Dutrow B (2007) Manual of Mineral Science (John Wiley \& Sons, Hoboken, NJ), 23rd Ed.

[30] Stock M, Davis R, de Mirandés E, Milton MJT (2019) The revision of the SI the result of three decades of progress in metrology. Metrologia 56(2):022001. https://doi.org/10.1088/1681-7575/ab0013

[31] Robinson IA, Schlamminger S (2016) The watt or Kibble balance: a technique for implementing the new si definition of the unit of mass. Metrologia 53:A46-A74. https://doi.org/10.1088/0026-1394/53/5/A46

[32] Holloway CL, Gordon JA, Jefferts S, Schwarzkopf A, Anderson DA, Miller SA, Thaicharoen N, Raithel G (2014) Broadband Rydberg atom-based electric-field probe for SI-traceable, self-calibrated measurements. IEEE Transactions on Antennas and Propagation 62(12):6169-6182. https://doi.org/10.1109/TAP.2014.2360208

[33] Holloway CL, Simons MT, Gordon JA, Wilson PF, Cooke CM, Anderson DA, Raithel G (2017) Atom-based RF electric field metrology: from self-calibrated measurement to subwavelength and near-field imaging. IEEE Transactions on Electromagnetic Compatibility 59(2):717-728. https://doi.org/10.1109/TEMC.2016.2644616 [20]

[34] Ehrlich CD, Rasberry SD (1998) Metrological timelines in traceability. fournal of Research of the National Institute of Standards and Technology 103(1):93-105. https://doi.org/10.6028/jres.103.005

[35] NIST (2019) NIST Quality Manual for Measurement Services (National Institute of Standards and Technology, U.S. Department of Commerce, Gaithersburg, Maryland). NIST QM-I Version 11 Available at https://www.nist.gov/ nist-quality-system/.

[36] Leaman DS, Hackett B (2020) National Voluntary Laboratory Accreditation Program - Procedures and General Requirements (National Institute of Standards and Technology, Gaithersburg, MD). https://doi.org/10.6028/NIST.HB.150-2020. NIST Handbook 150, 2020 Edition

[37] Lafarge T, Possolo A (2015) The NIST Uncertainty Machine. NCSLI Measure fournal of Measurement Science 10(3):20-27. https://doi.org/10.1080/19315775.2015.11721732 [27]

[38] Brand WA, Coplen TB, Vogl J, Rosner M, Prohaska T (2014) Assessment of international reference materials for isotope-ratio analysis (IUPAC Technical Report). Pure and Applied Chemistry 86:425-467. https://doi.org/10.1515/pac-2013-1023 